\title{
PKA/AKAP1 and PP2A/B $\beta 2$ Regulate Neuronal Morphogenesis via Drp1 Phosphorylation and Mitochondrial Bioenergetics
}

\author{
Audrey S. Dickey and Stefan Strack \\ Department of Pharmacology and Neuroscience Graduate Program, University of Iowa, Iowa City, Iowa 52242
}

\begin{abstract}
Mitochondrial shape is determined by fission and fusion reactions, perturbation of which can contribute to neuronal injury and disease. Mitochondrial fission is catalyzed by dynamin-related protein 1 (Drp1), a large GTPase of the dynamin family that is highly expressed in neurons and regulated by various posttranslational modifications, including phosphorylation. We report here that reversible phosphorylation of Drp1 at a conserved Ser residue by an outer mitochondrial kinase (PKA/AKAP1) and phosphatase (PP2A/B $\beta 2$ ) impacts dendrite and synapse development in cultured rat hippocampal neurons. PKA/AKAP1-mediated phosphorylation of Drp1 at Ser656 increased mitochondrial length and dendrite occupancy, enhancing dendritic outgrowth but paradoxically decreasing synapse number and density. Opposing PKA/AKAP1, PP2A/B $\beta 2$-mediated dephosphorylation of Drp1 at Ser656 fragmented and depolarized mitochondria and depleted them from dendrites, stunting dendritic outgrowth but augmenting synapse formation. Raising and lowering intracellular calcium reproduced the effects of dephospho-Drp1 and phospho-Drplon dendrite and synapse development, respectively, while boosting mitochondrial membrane potential with L-carnitine-fostered dendrite at the expense of synapse formation without altering mitochondrial size or distribution. Thus, outer mitochondrial PKA and PP2A regulate neuronal development by inhibiting and promoting mitochondrial division, respectively. We propose that the bioenergetic state of mitochondria, rather than their localization or shape per se, is the key effector of Drp1, altering calcium homeostasis to modulate neuronal morphology and connectivity.
\end{abstract}

\section{Introduction}

Mitochondria undergo fission/fusion processes that dynamically change their morphology in response to intrinsic and extrinsic signals. Dynamin-related protein 1 (Drp1) is a large GTPase that is recruited to the outer mitochondria membrane (OMM) by an as yet incompletely characterized adaptor protein complex, where Drp1 hydrolyzes GTP to induce OMM constriction and ultimately binary fission (Lackner and Nunnari, 2009; Westermann, 2010; Otera and Mihara, 2011). Drpl gene deletion in mice results in mid-gestational embryonic death (Ishihara et al., 2009; Wakabayashi et al., 2009), and hypomorphic mutations in Drp1 can cause fatal birth defects in humans (Waterham et al., 2007). Drp1 undergoes extensive post-translational modifications, including phosphorylation, sumoylation, and nitrosylation (Otera and Mihara, 2011). Calcineurin (protein phosphatase 2B) dephosphorylates Drp1 at a highly conserved Ser residue in the C-terminal GTPase effector domain (numbered 617, 637, 656 , etc. depending on species and splice variant) to promote

\footnotetext{
Received June 21, 2011; revised Aug. 31, 2011; accepted Sept. 14, 2011.

Author contributions: S.S. designed research; A.S.D. performed research; A.S.D. analyzed data; A.S.D. and S.S. wrote the paper.

This work was supported by NIH Grants NS043254, NS056244, NS057714 (S.S.), and American Heart Association predoctoral fellowship 0815519 G (A.S.D.). We thank Ron Merrill for comments on this manuscript and Yuriy Usachev for many helpful discussion.

Correspondence should be addressed to Stefan Strack, Department of Pharmacology, University of lowa, 51 Newton Road, lowa City, IA 52242. E-mail: stefan-strack@uiowa.edu.

DOI:10.1523/JNEUROSCI.3159-11.2011

Copyright $\odot 2011$ the authors $\quad 0270-6474 / 11 / 3115716-11 \$ 15.00 / 0$
}

mitochondrial fragmentation (Cribbs and Strack, 2007; Cereghetti et al., 2008). Protein kinase A (PKA)-mediated phosphorylation at the same site inhibits Drp1 to elongate mitochondria via unopposed fusion (Chang and Blackstone, 2007; Cribbs and Strack, 2007). PKA is targeted to the OMM by A kinase anchoring protein 1 (AKAP1, also known as D-AKAP1, AKAP121, AKAP149, and s-AKAP84) (Lin et al., 1995; Carlucci et al., 2008) enhancing Drp1 phosphorylation, mitochondrial elongation, and neuroprotection (Merrill et al., 2011). We previously showed that $\mathrm{B} \beta 2$, a neuron-specific regulatory subunit of protein phosphatase $2 \mathrm{~A}(\mathrm{PP} 2 \mathrm{~A})$ translocates to the OMM to cause mitochondrial fragmentation, although the mechanisms were not described (Dagda et al., 2003, 2005, 2008). PP2A exists predominantly as a heterotrimer of a catalytic $\mathrm{C}$, a scaffolding A, and a variable regulatory $B$ subunit. In mammals, 12 genes encode PP2A regulatory subunits thought to direct subcellular localization and substrate specificity of the heterotrimer (Janssens et al., 2008). The neurodegenerative disorder spinocerebellar ataxia type- 12 is caused by a noncoding CAG repeat expansion in the PPP2R2B gene, which encodes the cytosolic $\mathrm{B} \beta 1$ and the mitochondrial $\mathrm{B} \beta 2$ splice variants in humans (Holmes et al., 1999).

$\mathrm{B} \beta 2$ expression rises sharply during the first 2 weeks of postnatal brain development in rodents, a period of peak synaptogenesis (Dagda et al., 2003). Furthermore, Drp1-dependent mitochondrial fission has previously been shown to be important for synapse development and function (Li et al., 2004; Verstreken et al., 2005; Li et al., 2008). For these reasons, we sought to investigate the role of the mitochondrial fission/fusion regulators 

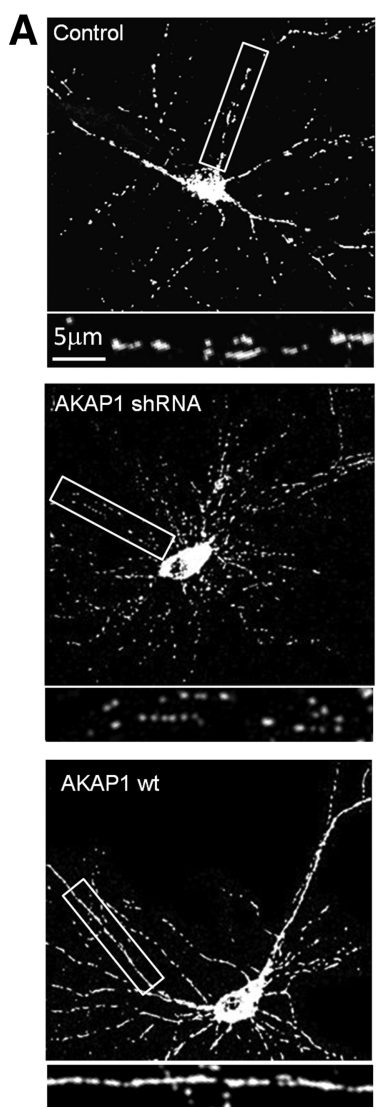

C

\section{B}
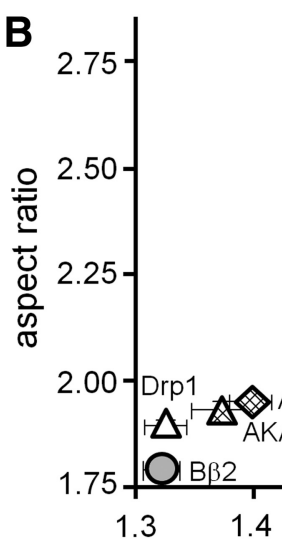
1.3
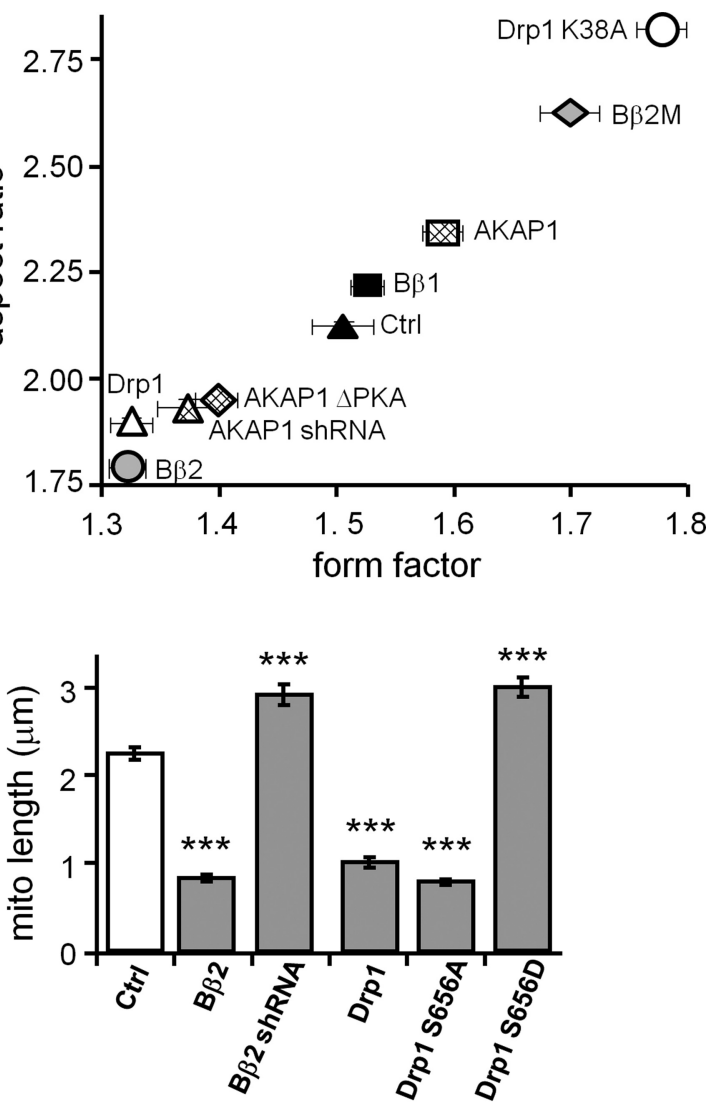

Figure 1. PKA/AKAP1 and phospho-Drp1 induce elongation, while PP2A/B $\beta 2$ and dephospho-Drp1 induce fragmentation of mitochondria. A-C, Hippocampal neurons cotransfected at 12-13 DIV with mitochondria-localized GFP, and the indicated constructs were fixed and imaged $4 \mathrm{~d}$ later. Representative confocal images. (white: mitochondria) are shown in $\boldsymbol{A}$. Morphology of dendritic mitochondria is quantified as aspect ratio and form factor in $\boldsymbol{B}$ (means $\pm \mathrm{SE}$ of 4 experiments, $45+$ neurons/condition), and as length in $\boldsymbol{C}$ (means \pm SE of three experiments, 36+ neurons/condition). AKAP1 $\triangle \mathrm{PKA}$, I310P, L316P mutant; B $32 \mathrm{M}$, RR168EE mutant; Ctrl, scrambled shRNA control; ${ }^{* * *} p<0.001$.

$\mathrm{PP} 2 \mathrm{~A} / \mathrm{B} \beta 2$ and PKA/AKAP1 in dendrite and synapse formation. Our results show that Drp1 dephosphorylation by PP2A/B $\beta 2$ causes mitochondrial fragmentation, depletes mitochondria from dendrites, and decreases their membrane potential $\left(\Delta \Psi_{\mathrm{m}}\right)$. This results in stunted dendritic outgrowth, but enhanced synapse formation. Opposing the OMM phosphatase, PKA/AKAP1 phosphorylates Drp1 at Ser656 to increase mitochondrial length, dendritic occupancy, and $\Delta \Psi_{\mathrm{m}}$, which increases dendrite complexity at the expense of synapse formation. We also provide evidence that mitochondrial membrane potential and calcium homeostasis mediate the effects of mitochondrial shape changes on neuronal development.

\section{Materials and Methods}

Antibodies and reagents. Commercial sources for antibodies and reagents used in these studies are as follows: PSD95 (postsynaptic density 95) (NeuroMab); bassoon (Stressgen); VGluT1 (vesicular glutamate transporter 1) (Millipore Bioscience Research Reagents); TOM20 (translocase of outer mitochondrial membrane $20 \mathrm{kDa}$ ) (Santa Cruz Biotechnology); EEA-1 (early endosome antigen 1) (Affinity Bioreagents); GFP (Abcam); MAP2B (microtubule-associated protein 2B) (BD Transduction Laboratories); Alexa Fluor dyes (488, 568, 647) (Invitrogen); BAPTA-AM (Invitrogen); BayK 8644 (Tocris Bioscience); and L-carnitine (Sigma).

Vectors. B $\beta 1$-GFP and wild-type and mutant $\mathrm{B} \beta 2$-GFP, mouse AKAP1-GFP, rat AKAP1-GFP (aa 1-524 of the N0 splice variant with C-terminal GFP), rat AKAP1 PKA-binding deficient mutant I310P, L316P (AKAP1 $\triangle$ PKA), rat wild-type Drp1, catalytically inactive Drp1 (K38A), Drp1 replacement constructs that express shRNA targeting en-

dogenous Drp1 while expressing either shRNA-resistant wild-type or phosphorylation site-mutant Drpl (S656A, S656D), and mitochondria-targeted GFP expression vectors were described previously, as were shRNAs against $\mathrm{B} \beta 2$ and AKAP1 (Cribbs and Strack, 2007; Dagda et al., 2008; Merrill et al., 2011). We expressed the brain-specific splice form of Drp1 that includes exons 3, 16, and 17 (GenBank accession no. AF019043) (Yoon et al., 1998). Lentivirus (feline immunodeficiency virus or FIV) expressing GFP-tagged Drp1, B $\beta 2$, and AKAP1 from a CMV promoter or shRNAs from an $\mathrm{H} 1$ promoter were generated by the University of Iowa Viral Vector Core (Iowa City, IA) and were described previously (Cribbs and Strack, 2007; Dagda et al., 2008; Merrill et al., 2011).

Preparation of dissociated hippocampal cultures. Primary cultures of hippocampal neurons were prepared from embryonic day 18 (E18) Sprague Dawley rats (Harlan) of either sex as described previously (Lim et al., 2003). Hippocampi were dissected and dura removed. Pooled tissue were incubated in HEPESbuffered saline (HBS; $10 \mathrm{~mm}$ HEPES, $150 \mathrm{~mm}$ $\mathrm{NaCl}, \mathrm{pH} 7.4, \mathrm{Ca}^{2+} / \mathrm{Mg}^{2+}$-free), containing trypsin $(0.03 \%)$ at $37^{\circ} \mathrm{C}$ for $20 \mathrm{~min}$. The tissues were washed three times with $\mathrm{Ca}^{2+} / \mathrm{Mg}^{2+}$-free HBS before cells were dissociated by trituration. Cells were plated in Neurobasal media (Invitrogen) containing NS21 supplement (Chen et al., 2008), glutamine ( $0.6 \mathrm{~mm})$, and $5 \%$ horse serum on poly-L-lysine-coated glass coverslips. Medium was changed to serum-free medium after $4 \mathrm{~h}$ of incubation. Cells were maintained at $37^{\circ} \mathrm{C}$ in a humidified environment of $95 \%$ air $/ 5 \% \mathrm{CO}_{2}$.

Fluorescence microscopy. Hippocampal cultures were washed with PBS before fixation with $4 \%$ paraformaldehyde for $10 \mathrm{~min}$. Fixed cells were washed, permeabilized with $0.1 \%$ Triton X-100, incubated in blocking solution (PBS with $2 \%$ donkey serum, $30-60 \mathrm{~min}$ ), and stained with antibodies against transfection/transduction markers (GFP) and various compartment marker proteins (i.e., TOM20, EEA-1, bassoon) for 4-16 h. After washes, cultures were incubated for $2 \mathrm{~h}$ with the appropriate Alexa Fluor dyeconjugated secondary antibodies (Invitrogen) and washed and mounted in Vectashield mounting medium before viewing. For analysis of mitochondrial morphology and localization, as well as synapse quantification, neurons were imaged on a Zeiss LSM 510 laser scanning confocal microscope with a $40 \times$ or $63 \times$ oil-immersion objective (University of Iowa Central Microscopy Research Facilities, Iowa City, IA). For analysis of dendrite complexity, a Leica DMI4000B epifluorescence microscope was used with a $20 \times$ or $40 \times$ air objective.

Mitochondrial morphology and localization. For analysis at 17 days in vitro (DIV), hippocampal cultures were transfected 4 to $5 \mathrm{~d}$ prior using Lipofectamine 2000 (0.08\% with $20 \mu \mathrm{g} / \mathrm{ml}$ plasmid). Plasmids encoding mitochondrial matrix-localized GFP (COX8 import sequence) or membrane-targeted mCherry ( $\mathrm{N}$ terminus of Lck, gift from Steven Green, Department of Biology, University of Iowa, Iowa City, IA) were included in the transfection mix at $10-20 \%$ of total plasmid mass to label mitochondria and processes, respectively. For analysis at 7 DIV, hippocampal cultures were transduced at 3 DIV with lentivirus. Cultures were fixed, stained, and imaged by confocal microscopy using constant settings for laser intensity, amplifier gain, and other parameters. Images were enhanced by low-pass filter and deconvolution (iterative 2D deconvolution plugin for ImageJ) before analysis. Mitochondrial morphology was quantified from auto-segmented images with an ImageJ macro reporting several measures including form factor $\left(\mathrm{FF}=\left(\right.\right.$ perimeter $\left.^{2}\right) /\left(4^{*}\right.$ 
$\pi^{*}$ area $)$ ), aspect ratio (AR $=$ major axis $/ \mathrm{mi}^{-}$ nor axis), and length as previously described (Cribbs and Strack, 2009; Merrill et al., 2011). Both FF and AR have a minimal value of 1 , which represents a perfect circle, with values increasing as mitochondria elongate. Autosegmented images were also analyzed for the number of mitochondria per dendrite length and the ratio of mitochondrial area to dendrite area using an ImageJ macro.

Dendrite outgrowth. Hippocampal cultures stained with MAP2B antibodies to label dendrites, but not axons, were subjected to Sholl analysis to quantify dendritic tree complexity. Dendrite intersections with $25 \mu \mathrm{m}$-spaced concentric circles starting at $50 \mu \mathrm{m}$ from the cell soma were counted as described previously (Zha et al., 2005).

Synapse development. Hippocampal cultures were transfected or virally transduced and fixed at 17 DIV. Some cultures were chronically treated with $1 \mu \mathrm{M}$ tetrodotoxin (TTX) and $100 \mu \mathrm{M}(2 R)$-amino-5-phosphonovaleric acid (APV) with fresh drug replacement every $3 \mathrm{~d}$ as described previously (Kossel et al., 1997; Rao and Craig, 1997; O’Brien et al., 1998; Turrigiano et al., 1998). To visualize synapses, cultures were double-labeled for a presynaptic marker (bassoon or VGluT1) and a postsynaptic marker (F-actin or PSD95). Confocal images were auto-segmented and presynaptic and postsynaptic overlap images were generated (AND function) using ImageJ software. Synaptic puncta were counted using the "Analyze particles" function of ImageJ.

Mitochondrial membrane potential. At 17-18 DIV, virally transduced hippocampal cultures were incubated with $20 \mathrm{~nm}$ tetramethylrhodamine methyl-ester (TMRM, Invitrogen) for $30 \mathrm{~min}$ at $37^{\circ} \mathrm{C}$ to allow dye equilibration across the plasma and inner mitochondrial membranes (Farkas et al., 1989; Scaduto and Grotyohann, 1999; Verburg and Hollenbeck, 2008). Mitochondria were imaged live by confocal microscopy. Cultures were treated for 10 min with $0.5 \mu \mathrm{M}$ FCCP (carbonyl-cyanide $p$-(trifluoro-methoxy) phenylhydrazone) and $2 \mu \mathrm{M}$ oligomycin or $1 \mathrm{~mm} \mathrm{~L}$-carnitine to decrease or increase, respectively, mitochondrial membrane potential, $\Delta \Psi_{\mathrm{m}}$. TMRM fluorescence $(\mathrm{Fl})$ ratios were calculated according to the formula $\left(\mathrm{Fl}_{\text {mito }}-\mathrm{Fl}_{\text {back }}\right) /\left(\mathrm{Fl}_{\text {cyt }}-\mathrm{Fl}_{\text {back }}\right)$ (Scaduto and Grotyohann, 1999; Verburg and Hollenbeck, 2008).

Statistical analysis. Data were analyzed by two-tailed Student's $t$ test for single comparisons and by one-way ANOVA followed by pairwise Bonferroni post hoc tests for multiple comparisons. All analyses were performed blind to the experimental conditions.

\section{Results}

AKAP1-anchored PKA and

$\mathrm{B} \boldsymbol{\beta} 2$-targeted $\mathrm{PP} 2 \mathrm{~A}$ regulate

mitochondrial shape in dendrites

We began by comparing the effects of manipulating PKA/ AKAP1, PP2A/B $\beta 2$, and Drp1 activity on mitochondrial shape in cultured hippocampal neurons. Mitochondria were visualized by cotransfection with matrix-targeted GFP [cytochrome oxidase VIII (COX8) import sequence]. Compared to two controls, scrambled shRNA and cytososolic PP2A/B $\beta 1$, transfection of Drp1 and B $\beta 2$ caused similar mitochondrial fragmentation, while dominant-
C

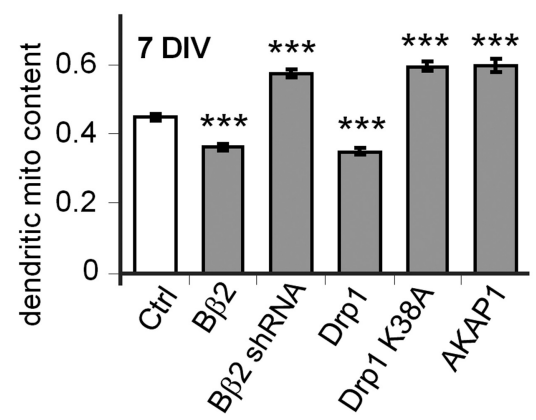

D

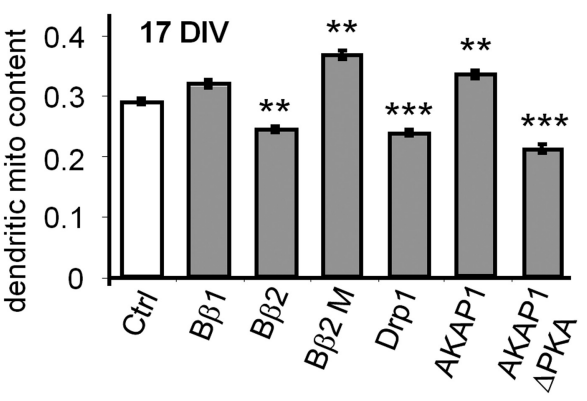

E

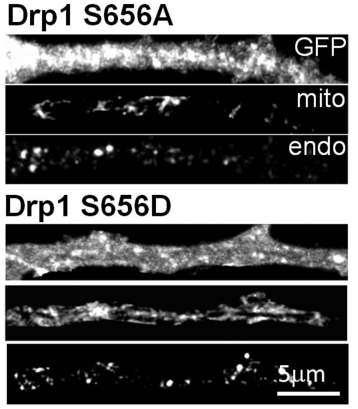

$\mathbf{F}$

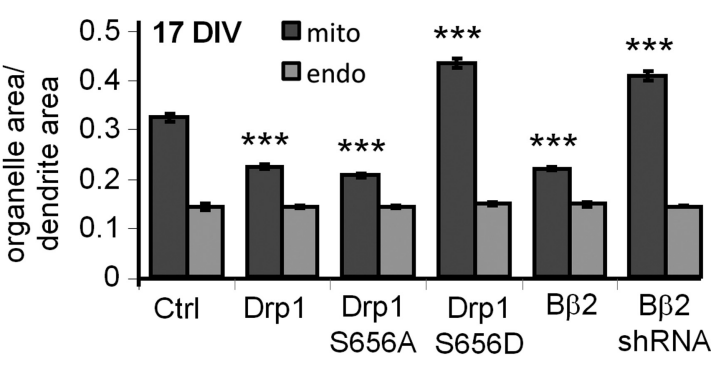

Figure 2. $\mathrm{PP} 2 \mathrm{~A} / \mathrm{B} \beta 2$ and dephospho-Drp1 decrease, while PKA/AKAP1 and phospho-Drp1 increase dendritic mitochondria (mito) at 7 ( $\boldsymbol{C}$ or 17 DIV $(\boldsymbol{A}, \boldsymbol{B}, \boldsymbol{D})$. Representative confocal images of primary dendrites are shown in $\boldsymbol{A}$. Dendritic mitochondria content (ratio of

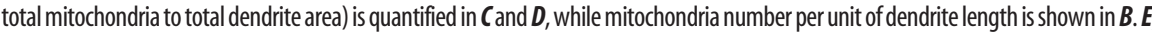
fixed at 17 DIV and stained for mitochondria (TOM20) and endosomes (EEA1). $\boldsymbol{E}$ shows representative images of primary dendrites, and $\boldsymbol{F}$ shows total mitochondria and endosome (endo) area over primary dendrite area. Bar graphs show means \pm SE of three experiments with $45+(\boldsymbol{B}, \boldsymbol{D})$ and 36+ $(\boldsymbol{C}, \boldsymbol{F})$ neurons/condition; ${ }^{*} p<0.05,{ }^{* *} p<0.01,{ }^{* * *} p<0.001$. Ctrl, Control.

negative Drp1 (GTPase-deficient K38A mutant) and dominantnegative $\mathrm{B} \beta 2$ (PP2A holoenzyme binding-deficient, RR168EE mutant; Dagda et al., 2003) promoted mitochondrial elongation to the same extent (Fig. $1 B$, expressed as aspect ratio and form factor). Recruiting PKA to the OMM by overexpression of AKAP1 caused mitochondrial elongation, while efficient knockdown of AKAP1 with shRNA (Merrill et al., 2011) or expression of a AKAP1 mutant that does not bind PKA (AKAP1 I310P, L316P = $\triangle$ PKA; Affaitati et al., 2003; Merrill et al., 2011) induced mitochondrial fragmentation, the latter in an apparent dominant-negative manner (Fig. 1A, $B$ ).

We previously showed that PKA/AKAP1 fuses mitochondria by phosphorylating Drp1 at a conserved PKA site at the $\mathrm{N}$-terminal border of the GED (GTPase effector domain) (Merrill et al., 2011). This serine is at position 656 in the most abundant splice variant in the rat brain (Yoon et al., 1998), but is also referred in the literature as Ser600, 617, and 637, depending on species and splice variant. To examine the influence of Drp1 phosphorylation on mitochondrial morphology in hippocampal neuron dendrites, endog- 
A
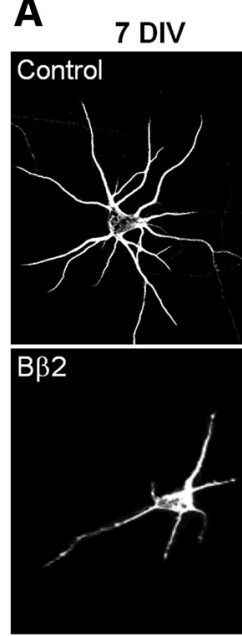

$B \beta 2$

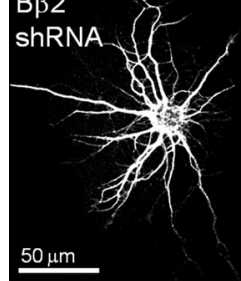

17 DIV

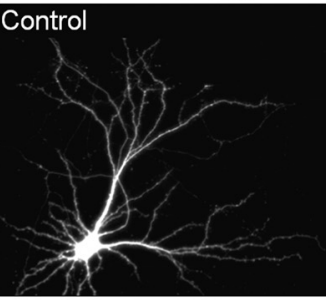

$\mathrm{B} \beta 2$

B

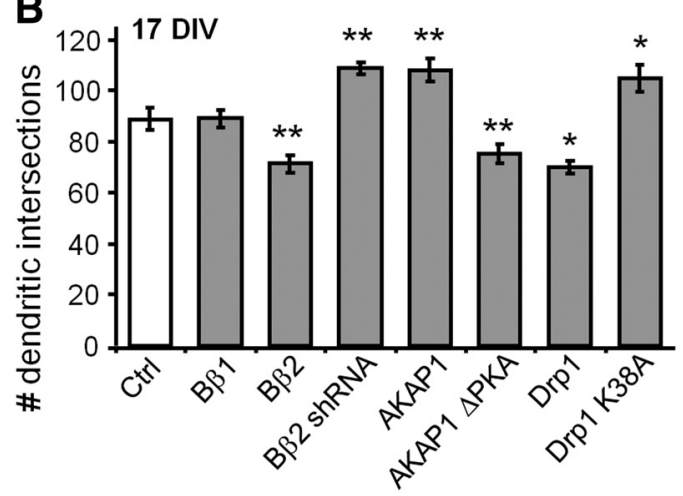

C
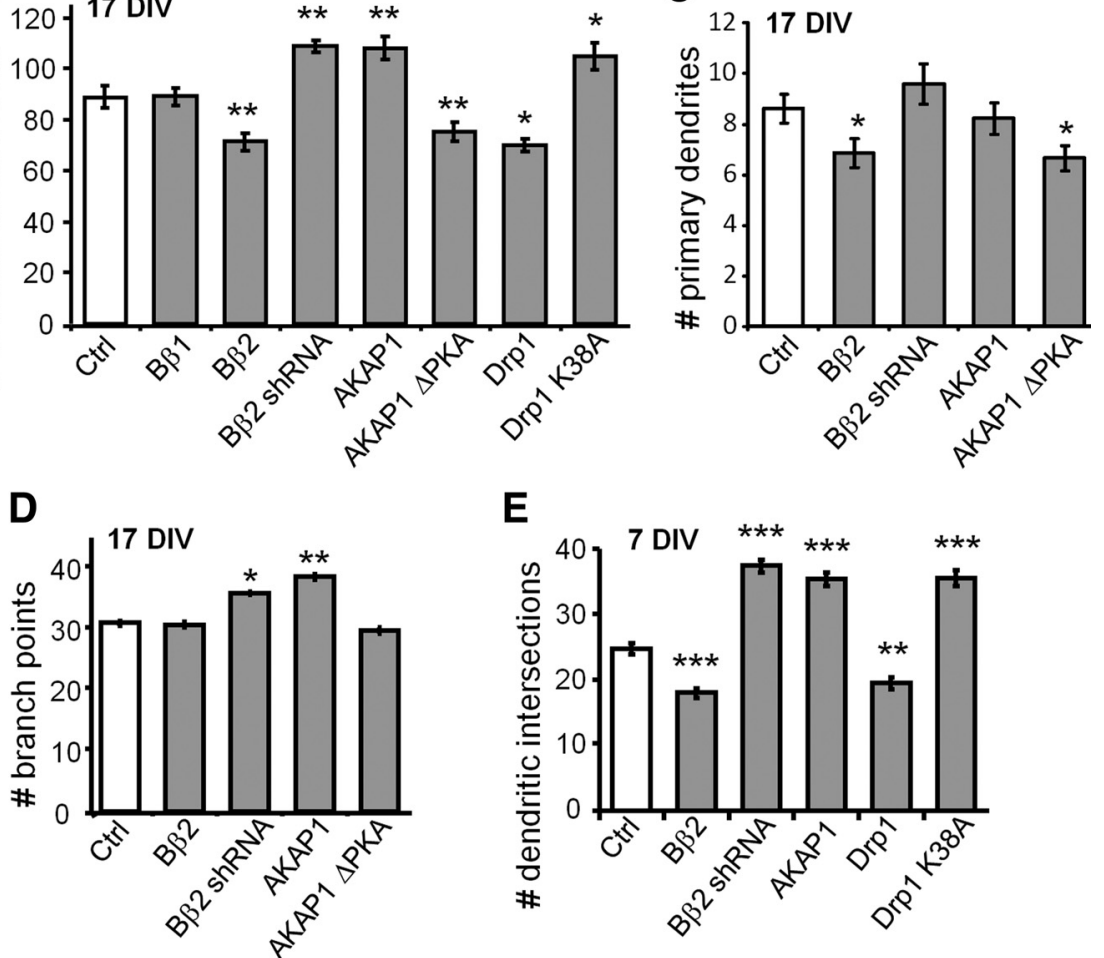

E

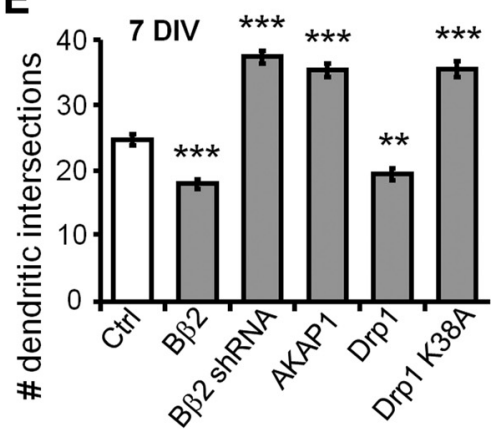

Figure 3. PKA/AKAP1 fosters while PP2A/B $\beta 2$ retards dendrite outgrowth. $\boldsymbol{A}-\boldsymbol{E}$, Hippocampal neurons virally transduced at 0 or 7 DIV were fixed and labeled for MAP2B at $7(\boldsymbol{A}, \boldsymbol{E})$ or 17 DIV $(\boldsymbol{A}-\boldsymbol{D})$; representative images are shown in $\boldsymbol{A}$. Dendrite complexity was quantified by Sholl analysis as the number of dendritic intersections with $25 \mu \mathrm{m}$-spaced concentric circles centered on the cell body $(\boldsymbol{B}, \boldsymbol{E})$ and by counting primary dendrites ( $C$ and dendritic branch points per neuron (D). Bar graphs show means \pm SE of $3-5$ experiments with $36+$ neurons $/$ condition; ${ }^{*} p<0.05,{ }^{* *} p<0.01,{ }^{* * *} p<0.001$. (trl, Control.

enous Drp1 was replaced with dephospho-Drp1 (S656A mutant) or pseudophosphorylated Drp1 (S656D mutant) by expressing Drp1directed shRNA and RNAi-resistant mutant Drp1 cDNAs from the same plasmid (Cribbs and Strack, 2007). Phospho-Drp1 increased mitochondrial length, while dephospho-Drp1 shortened mitochondria compared to empty vector transfection. $\mathrm{B} \beta 2$ overexpression and knockdown mimicked the phenotype of dephospho-Dp1 and phospho-Drp1, respectively (Fig. 1C), suggestive of an enzyme-substrate relationship.

\section{OMM-targeted PKA and PP2A regulate mitochondrial content in dendrites}

The fission/fusion equilibrium has previously been shown to influence mitochondrial transport in axons and dendrites ( $\mathrm{Li}$ and Sheng, 2003; Li et al., 2004; Verstreken et al., 2005; Baloh et al., 2007; Misko et al., 2010). We therefore investigated the role of PP2A- and PKAmediated mitochondrial restructuring in mitochondrial localization in dendrites. To this end, we counted dendritic mitochondria (labeled with COX8-GFP) and calculated dendritic mitochondria content as the ratio of cumulative mitochondrial area over total dendrite area (marked with membrane-targeted (Lck) mCherry). Overexpression of mitochondria-fragmenting $\mathrm{B} \beta 2$, but not cytosolic $\mathrm{B} \beta 1$, roughly doubled the number of dendritic mitochondria. Conversely, AKAP1-transfected neurons had fewer mitochondria in dendrites, while neurons expressing the PKA binding-deficient AKAP1 mutant had more than control (Fig. $2 A, B$ ). Because of the mitochondrial length changes associated with the mitochondrial fission regulators, total mitochondrial content in dendrites increased upon mitochondrial elongation following AKAP1 overexpression, shRNA-mediated silencing of $\mathrm{B} \beta 2$, or dominant-negative inhibition of $\mathrm{B} \beta 2(\mathrm{~B} \beta 2 \mathrm{M})$ and Drp1 (K38A). Fragmenting mitochondria by transfection of dominant-negative AKAP1 ( $\triangle \mathrm{PKA})$, wild-type $\mathrm{B} \beta 2$, or Drp1 conversely lowered mitochondrial occupancy in dendrites. Similar effects were observed in immature (7 DIV) and mature (17 DIV) cultures (Fig. 2C,D), suggesting that synapses are not necessary for this effect.

To gain further evidence that PKA and PP2A-mediated changes in mitochondrial content in dendrites are specific and involve Drp1 phosphorylation, transfected hippocampal neurons were immunofluorescently labeled for mitochondria (TOM20) and early endosomes (EEA-1). Silencing of PP2A/B $\beta 2$ and pseudo-phosphorylation of Drp1 (replacement of endogenous with S656D mutant) resulted in similar increases in mitochondria content in primary dendrites, whereas overexpression of Drp1 and $\mathrm{B} \beta 2$, as well as replacement of endogenous with dephospho-Drp1 (S656A), had the opposite effect (Fig. 2E,F). Total endosome area over dendrite area did not change with any of the transfections $(\sim 0.15$; Fig. $2 E, F)$, arguing against effects on organelle transport in general.

\section{$\mathrm{PKA} / \mathrm{AKAP} 1$ and $\mathrm{PP} 2 \mathrm{~A} / \mathrm{B} \boldsymbol{\beta} 2$ regulate dendritogenesis}

Coinciding with the major period of neurite outgrowth and synaptogenesis, $\mathrm{B} \beta 2$ expression increases from undetectable levels at birth to plateau at postnatal day 14 in the rat CNS (Dagda et al., 2003). $\mathrm{B} \beta 2$ is highly expressed in forebrain regions, including cortex and hippocampus, but is undetectable in non-neuronal tissues (Schmidt et al., 2002; Dagda et al., 2003). To explore a possible role of $\mathrm{PP} 2 \mathrm{~A} / \mathrm{B} \beta 2$ and its opposing kinase PKA/AKAP1 in dendrite development, transfected hippocampal cultures were subjected at 17 DIV to Sholl analysis (counting the number of MAP2B-positive dendrite intersections with concentric circles centered over the soma). We found that $\mathrm{B} \beta 2$ is a negative regulator of dendrite outgrowth, with overexpression decreasing and 

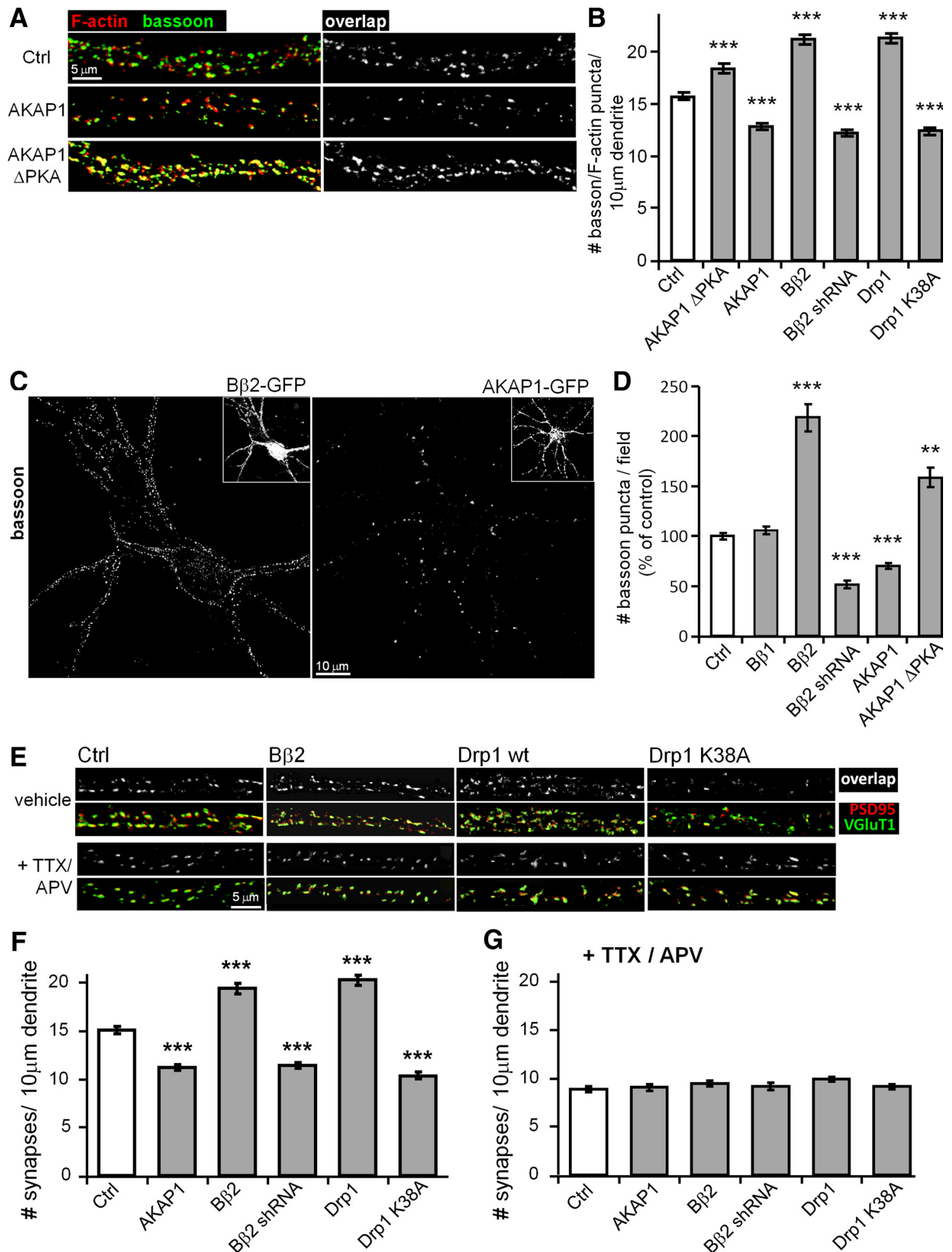

Figure 4. Mitochondrial fission promotes activity-dependent synapse formation. $A-G, H C$ neurons were virally transduced at 3 DIV and fixed at $17-18$ DIV. In G, excitatory neurotransmission was blocked with $\Pi \mathrm{TX}(1 \mu \mathrm{M})$ and APV $(100 \mu \mathrm{m})$ throughout this period. Synapses were identified by immunolabeling for presynaptic and postsynaptic markers, bassoon/F-actin $(\boldsymbol{A}-\boldsymbol{D})$ and vGluT1/PSD95 $(\boldsymbol{E}, \boldsymbol{F})$, and regions of overlap $(\boldsymbol{A}, \boldsymbol{D}, \boldsymbol{E}, \boldsymbol{F})$ or presynaptic puncta $(\boldsymbol{C}, \boldsymbol{D})$ were counted by automated image analysis. Numbers of presynaptic, postsynaptic, and overlap puncta were essentially identical for each neuron. Representative confocal images of primary dendrites $(\boldsymbol{A}, \boldsymbol{E})$ and full dendritic arbors $(\boldsymbol{C})$ are shown. Synapse density (number per $10 \mu \mathrm{m}$ primary dendrite) is plotted in $\boldsymbol{B}, \boldsymbol{F}, \boldsymbol{G}$, while total synapse number (per $150 \times 150 \mu \mathrm{m}$ visual field) is shown in $\boldsymbol{D}$ as means \pm SE of $3-4$ experiments with $36+$ neurons/condition; ${ }^{*} p<0.05,{ }^{* *} p<0.01$, ${ }^{* * *} p<0.001$, compared to control (Ctrl).

RNAi-mediated knockdown increasing dendrite complexity (Fig. $3 A, B$ ).

AKAP1 overexpression enhanced dendrite outgrowth to the same extent as B $\beta 2$ RNAi, while the AKAP1 $\triangle$ PKA mutant phenocopied $B \beta 2$ overexpression (Fig. $3 B$ ). Implicating the mito- chondrial fission enzyme as the key effector, overexpression of wild-type Drp1 mimicked the effect of the mitochondriafragmenting phosphatase, whereas dominant-negative Drp1 (K38A) stimulated dendrite outgrowth similarly to AKAP1 overexpression or $\mathrm{B} \beta 2$ knockdown (Fig. $3 B$ ). 
Primary dendrites and dendritic branch points were counted in 17 DIV neurons transfected with a subset of constructs. Mitochondrial fragmentation via expression of $\mathrm{B} \beta 2$ or AKAP1 $\triangle \mathrm{PKA}$ was associated with a significant decrease in the number of primary dendrites, while neurons expressing B $\beta 2$ shRNA displayed a trend toward more primary dendrites (Fig. $3 C$ ). As for dendritic branching, mitochondrial elongation by $\mathrm{B} \beta 2$ knockdown or AKAP1 overexpression resulted in a small but significant increase in the number of branch points (Fig. 3D). Thus, mitochondrial shape influences dendrite initiation, branching, and elongation.

Dendrite development is modulated by synaptic activity (Vaillant et al., 2002; Van Aelst and Cline, 2004). We transduced hippocampal cultures within $3 \mathrm{~d}$ after plating and analyzed dendrite complexity in immature neurons that lack detectable synapses. Results from Sholl analysis in these 7 DIV cultures mirrored those from mature 17 DIV cultures, with mitochondrial fission-inducing plasmids stunting and fusion-promoting plasmids enhancing dendritogenesis, except that effect amplitudes were greater in younger than in older neurons (compare Fig. 3, $B$ and $E$ ). These results indicate that kinase/phosphatase-regulated mitochondrial morphogenesis impinges on early process outgrowth, as distinguished from dendrite pruning and other activity-dependent mechanisms in mature neurons.

\section{$\mathrm{PKA} / \mathrm{AKAP} 1$ and $\mathrm{PP} 2 \mathrm{~A} / \mathrm{B} \boldsymbol{\beta} 2$ regulate synaptogenesis}

Drp1 overexpression and dominant-negative inhibition were previously shown to promote and inhibit, respectively, dendritic spine formation in hippocampal cultures (Li et al., 2004, 2008). To extend these findings to OMM-targeted PKA, PP2A, and (de)phospho-Drp1, we visualized innervated dendritic spines by two methods. First, hippocampal cultures transfected at 3 DIV were costained at 17-18 DIV for the dendritic spine marker F-actin and the presynaptic terminal marker bassoon. Areas of overlap between F-actin and bassoon or bassoon puncta alone were counted as synapses by automated image analysis. Essentially identical results were obtained by costaining cultures for the postsynaptic density protein PSD95 and the vesicular glutamate transporter VGluT1 as a presynaptic marker and quantifying excitatory synapses by counting puncta of label coincidence as in the first method. Hippocampal cultures were transfected with plasmids or transduced with lentivirus to no more than 5\% efficiency, such that most presynaptic terminals analyzed were derived from naive neurons. Therefore, effects on synaptogenesis originated postsynaptically from the transfected/transduced neuron. Only data from primary dendrites are reported here; however, we obtained essentially identical results when quantifying synapse density in secondary and tertiary dendrites (data not shown).

We found that mitochondrial fission/fusion regulators impact dendritogenesis and synaptogenesis in opposite directions. Inducing mitochondrial fission (Drp1 or $\mathrm{B} \beta 2$ overexpression, AKAP1 $\triangle$ PKA) led to an increase in both synapse density (number per $10 \mu \mathrm{m}$ primary dendrite; Fig. $4 A, B, E-G$ ) and synapse number per neuron (within a 150 by $150 \mu \mathrm{m}$ visual field; Fig. $4 C, D)$. On the other hand, unopposed mitochondrial fusion (dominant-negative Drp1 K38A, AKAP1 overexpression, B $\beta 2$ silencing) decreased synapse density and total number. Indeed, changes in synapse number per visual field were of larger amplitude than changes in synapse density on primary dendrites $( \pm 100 \%$ vs $\pm 30 \%)$, because synapse density tapered off more steeply distal from the soma in neurons with more complex dendritic arbors (B $\beta 2$ shRNA, AKAP1 overexpression).
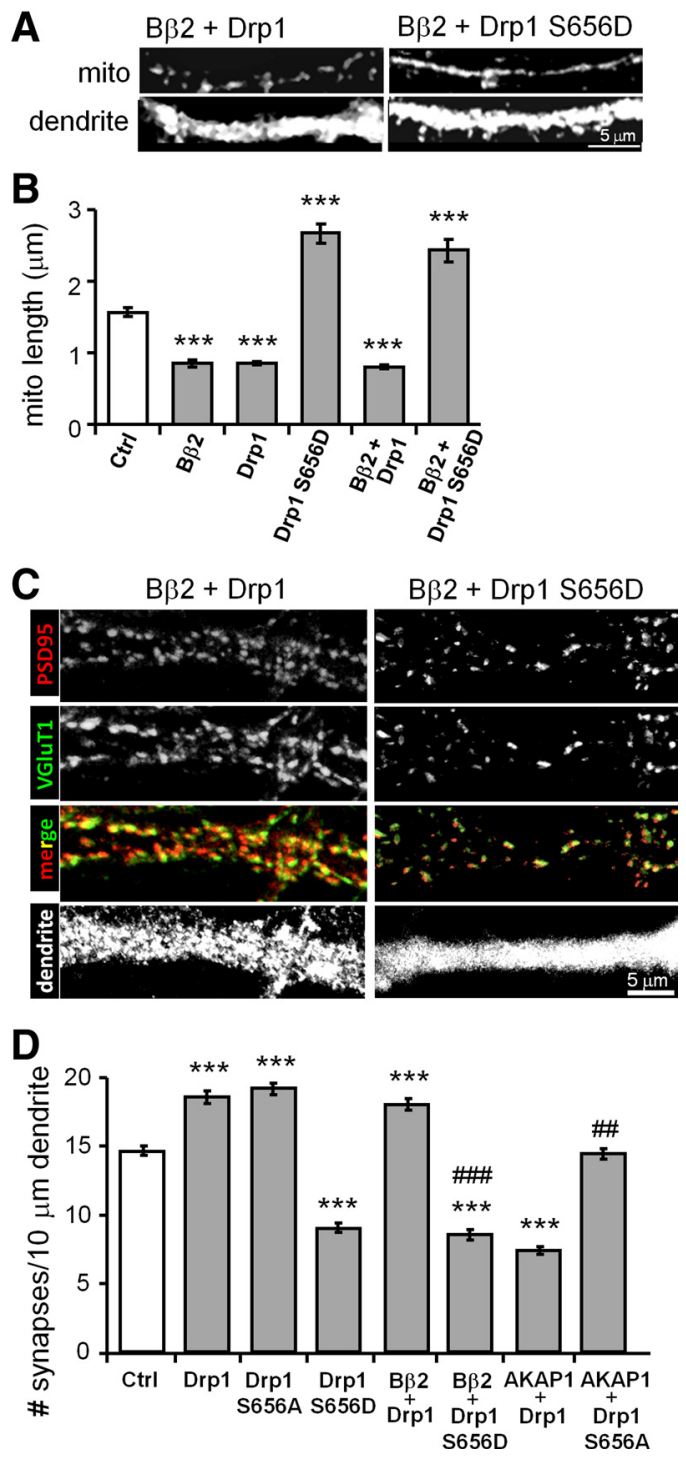

Figure 5. PP2A/B $\beta 2$ and PKA/AKAP1 act through (de)phosphorylation of Drp1 at Ser656. $\boldsymbol{A}-\boldsymbol{D}$, Hippocampal neurons transfected at 13 DIV were fixed at 18 DIV and immunolabeled for mitochondria (mito, TOM20; $\boldsymbol{A}, \boldsymbol{B}$ ) or glutamatergic synapses (vGluT1 + PSD95, $\boldsymbol{C}, \boldsymbol{D}$ ). Representative confocal images of primary dendrites are shown in $\boldsymbol{A}$ and $\boldsymbol{C}$, while mitochondrial length and synapse density are plotted in $\boldsymbol{B}$ and $\boldsymbol{D}$, respectively, as means \pm SE of three experiments with $36+$ neurons/condition; ${ }^{*} p<0.05$, ${ }^{* *} p<0.01$, ${ }^{* * *} p<0.001$, compared to control (Ctrl); ${ }^{\# \#} p<0.1,{ }^{\# \#} p<0.001$, compared to AKAP1/B $\beta 2+$ Drp1.

Since mitochondria regulate calcium homeostasis (Nicholls et al., 2003; Pivovarova and Andrews, 2010) and, since calcium influx is required for synaptogenesis (Kossel et al., 1997), we inhibited excitatory $\mathrm{Ca}^{2+}$ influx in response to spontaneous synaptic activity by chronic $(\sim 14 \mathrm{~d})$ incubation of cultures with TTX ( 1 $\mu \mathrm{M})$ and APV $(100 \mu \mathrm{M})$ to block voltage-gated sodium channels and NMDA receptors, respectively. This treatment regimen was previously shown to decrease dendritic spine density in hippocampal cultures (Kossel et al., 1997).

We found that blocking synaptic transmission with TTX/APV abolished the stimulatory effect of Drp1 and PP2A/B $\beta 2$ overexpression on synaptogenesis, reducing synapse density to levels below control (Fig. 4E-G). Also in agreement with the literature (Kossel et al., 1997), blocking transmission had no effect on dendrite complexity under any of the transfection conditions tested, likely because dendrites are already fairly elaborate by the time 
synapses start to form in these cultures (data not shown). These results indicate that mitochondrial fission increases synapse number by a distinct, activitydependent mechanism, as opposed to through cellular adaptation to a primary effect on dendrite outgrowth.

\section{PKA/AKAP1 and PP2A/B $\beta 2$ act through Drp1 Ser656}

We previously showed that PKA/AKAP1 promotes neuronal survival by inhibiting Drp1 through phosphorylation at Ser656 (Merrill et al., 2011). While Drp1 K38A blocks $\mathrm{B} \beta 2$ overexpression-induced mitochondrial fission (Dagda et al., 2008), a different phosphatase, calcineurin (also known as PP2B) was previously implicated in the dephosphorylation and activation of Drp1 (Cribbs and Strack, 2007; Cereghetti et al., 2008). To examine whether $\mathrm{PP} 2 \mathrm{~A} / \mathrm{B} \beta 2$-mediated mitochondrial fragmentation and enhanced synaptogenesis requires Drpl's conserved PKA site, we transiently transfected hippocampal cultures with $\mathrm{B} \beta 2$ overexpression and Drp1 replacement vectors. Coexpression of $\mathrm{B} \beta 2$ and wild-type Drp1 decreased the length of dendritic mitochondria by $50 \%$, similar to transfection with either plasmid alone (Fig. $5 A, B$ ). This nonadditivity is evidence of $\mathrm{B} \beta 2$ and Drp1 operating in the same pathway. However, replacement of endogenous with pseudophosphorylated Drp1 (S656D) completely blocked the effect of phosphatase overexpression, elongating mitochondria beyond control levels (Fig. 5A,B).

In similar epistasis experiments with synapse quantification as an endpoint, pseudophosphorylation of Drp1 was found to reduce synapse density to $50 \%$ of control values. Moreover, Drp1 S656D replacement was dominant, eliminating the synaptogenic activity of coexpressed $\mathrm{B} \beta 2$ (Fig. $5 C, D$ ). Conversely, rendering Drp1 nonphosphorylatable raised synapse density to the same level as overexpressing Drp1. Also, Drp1 S656A blocked the synapse-thinning effect of recruiting PKA to the OMM via AKAP1 expression (Fig. 5D). In aggregate, these findings indicate that Ser656 in Drp1 is the key residue through which outer mitochondrial forms of PKA and PP2A modulate neuronal interconnectivity.

\section{PKA/AKAP1 and PP2A/B $\beta 2$ modulate mitochondrial membrane potential}

To begin to address the mechanism by which mitochondrial dynamics affects the development of hippocampal neurons in culture, we assessed $\Delta \Psi_{\mathrm{m}}$, mitochondrial membrane potential. AKAP1 has been reported to boost $\Delta \Psi_{\mathrm{m}}$ and mitochondrial ATP synthesis in a human embryonic kidney cell line (HEK293 cells; Livigni et al., 2006), whereas displacement of AKAP1 from mitochondria decreases $\Delta \Psi_{\mathrm{m}}$ in cultured smooth muscle cells (Perrino et al., 2010). Additionally, forced expression of Drp1 and Fis1 (an OMM-anchored profission protein) was shown to depolarize mitochondria in adult mouse muscle in vivo (Romanello et al., 2010).
Hippocampal neurons virally transduced with GFP fusion proteins (or GFP alone) were incubated with the $\Delta \Psi_{\mathrm{m}}$-sensitive fluorescent dye TMRM (20 nM), and primary dendrite segments were imaged live by confocal microscopy. As a voltage-dependent dye, TMRM rapidly accumulates in neurons where it equilibrates between cytosol and mitochondria according to the Nernst equation. Figure $6 B$ shows the data as the ratio of mitochondrial to cytosolic TMRM fluorescence. To establish a lower bound, we collapsed the mitochondrial proton gradient with $0.5 \mu \mathrm{M}$ FCCP in the presence of oligomycin $(2 \mu \mathrm{M})$ to prevent ATP depletion due to reversal of the ATP synthase (Verburg and Hollenbeck, 2008). As a positive control, $\Delta \Psi_{\mathrm{m}}$ was boosted by treating cultures with $1 \mathrm{~mm}$ L-carnitine, a metabolic intermediate that mediates matrix import of fatty acids for $\beta$-oxidation and mitochondrial respiration (Bremer, 1983; Yano et al., 2010).

Mitochondrial elongation through forced expression of AKAP1 or knockdown of $\mathrm{B} \beta 2$ hyperpolarized mitochondria significantly, although not to the same level as L-carnitine (Fig. 6A, B). Conversely, mitochondrial fission in response to overexpression of Drp1 or $\mathrm{B} \beta 2$ decreased $\Delta \Psi_{\mathrm{m}}$ to levels intermediate between GFP-expressing untreated and FCCP/oligomycin-treated neurons. Thus, in hippocampal neurons, just as in non-neuronal cells, mitochondrial membrane potential scales with length.

\section{Calcium manipulations alter dendrite outgrowth}

Mitochondrial membrane potential determines ATP synthesis, reactive oxygen species (ROS) generation, but also provides the 
A 19 DIV Control $\left(1.8 \mathrm{mM} \mathrm{Ca}^{2+}\right)$

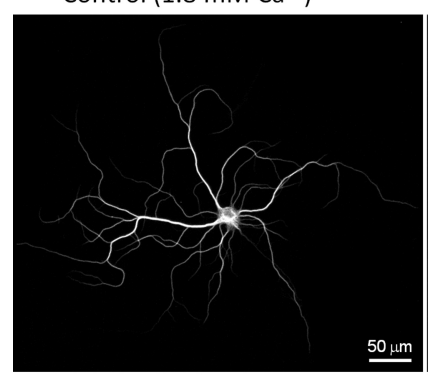

$2.3 \mathrm{mM} \mathrm{Ca}^{2+}$

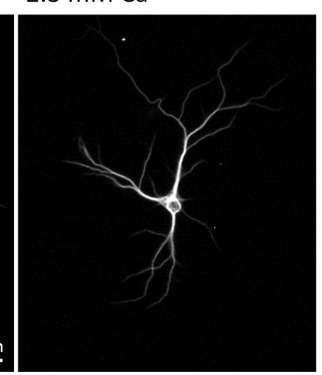

C 15 DIV

Control

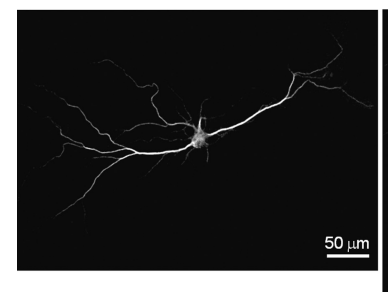

BAPTA-AM

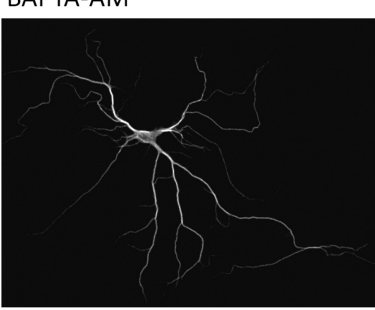

B

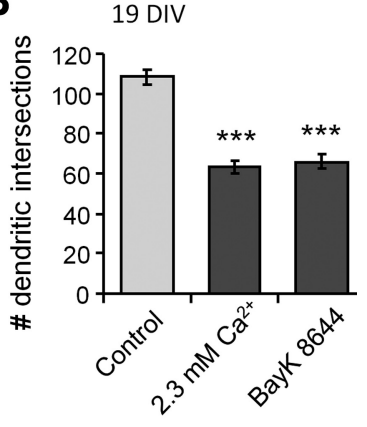

D

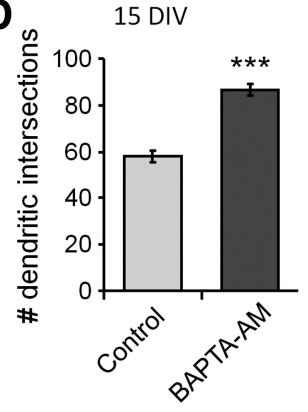

Figure 7. Calcium inhibits dendritogenesis. $\boldsymbol{A}-\boldsymbol{D}$, Hippocampal neurons were cultured in the presence of elevated calcium (2.3 $\mathrm{mM})$, the L-type $\mathrm{Ca}^{2+}$ channel agonist Bay K8466 (50 nm), or the cell-permeant calcium chelator BAPTA-AM (10 $\left.\mu \mathrm{m}\right)$ and fixed and stained for MAP2B at 15 or 19 DIV as indicated. Representative epifluorescence images are shown in $\boldsymbol{A}$ and $\boldsymbol{C}$, while results from Sholl analyses are plotted in $\boldsymbol{B}$ and $\boldsymbol{D}$ as means \pm SE of three experiments with $36+$ neurons/condition; ${ }^{* * *} p<0.001$.

driving force for sequestration of cytosolic calcium (Nicholls et al., 2003; Pivovarova and Andrews, 2010). Initial evidence that calcium may be the critical variable derived from experiments showing that $\mathrm{B} \beta 2$ and Drp1-induced synaptogenesis requires excitatory synaptic transmission (Fig. $4 E-G$ ). To further implicate calcium-dependent processes in mitochondrial control of neuronal morphogenesis, we performed Sholl analysis on hippocampal neurons cultured under conditions designed to raise or lower $\left[\mathrm{Ca}^{2+}\right]_{\mathrm{i}}$. To elevate $\left[\mathrm{Ca}^{2+}\right]_{\mathrm{i}}$, culture media was either supplemented to $2.3 \mathrm{mM} \mathrm{Ca}^{2+}$ (from normally $1.8 \mathrm{~mm}$ ), or the L-type calcium channel agonist BayK 8644 was added (50 nM) for $5 \mathrm{~d}$ before imaging MAP2B-stained processes at 19 DIV. Both treatments attenuated dendrite development by close to $50 \%$ (Fig. $7 A, B)$. In a second set of experiments, the cell-permeant calcium chelator BAPTA-AM $(10 \mu \mathrm{M})$ or vehicle was added at 12 DIV, and dendritic complexity was quantified at 15 DIV. Chelating intracellular $\mathrm{Ca}^{2+}$ caused exuberant dendrite outgrowth (50\% increase; Fig. $7 C, D)$. Suggestive of a causal link, mitochondrial fission and calcium thus exert complex but parallel effects on neuronal morphogenesis, impairing dendritogenesis but fostering synaptogenesis.

\section{L-Carnitine phenocopies effects of mitochondrial fusion on neuronal morphogenesis}

Lastly, we explored whether mitochondrial bioenergetics may be sufficient to explain how PKA/AKAP1, PP2A/B $\beta 2$, and Drp1 regulate neuronal morphogenesis. To this end, we used L-carnitine, a positively charged amino acid that promotes lipid metabolism by shuttling long-chain fatty acids into the mitochondrial matrix (Bremer, 1983). L-Carnitine was supplied throughout the culture period at $1 \mathrm{~mm}$, a concentration that robustly elevates oxidative phosphorylation as evidenced by increased mitochondrial TMRM fluorescence (Fig. 6A,B).
Neither the length of mitochondria nor their occupancy in dendrites was affected by L-carnitine (Fig. $8 A-C$ ). Nevertheless, $\mathrm{L}$-carnitine altered neuronal shape and connectivity as though mitochondria were elongated by AKAP1 overexpression, B $\beta 2$ knockdown, or Drp1 inhibition. Specifically, L-carnitine increased dendrite complexity (Fig. 8D,E) but decreased synapse number and density (Fig. $8 F-H)$. In addition, the metabolism booster "rescued" neurons from $\mathrm{B} \beta 2$ overexpression, overcoming dendrite stunting and eliminating supernumerary synapses (Fig. $8 E, H$ ).

\section{Discussion}

Figure 9 summarizes the major findings in this study. Briefly, we found that the mitochondrial fission/fusion equilibrium in hippocampal neurons is regulated by the opposing activities of an OMM-targeted kinase and a phosphatase. PKA/AKAP1 and $\mathrm{PP} 2 \mathrm{~A} / \mathrm{B} \beta 2$ converge on a single, highly conserved serine residue in the mitochondrial fission enzyme Drp1 to exert powerful but opposite effects on dendrite and synapse development. Mitochondrial fission induced by dephospho-Drp1 yielded shorter dendrites with more synapses, whereas phospho-Drp1 resulted in unopposed fusion, supporting dendrite at the expense of synapse development. Pointing to calcium as a critical effector, we found that blocking excitatory neurotransmission inhibits fissioninduced synaptogenesis, while intracellular calcium chelation enhanced early neurite outgrowth. Hyperpolarizing mitochondria with L-carnitine mirrored both effects of unopposed mitochondrial fusion without altering mitochondrial shape or localization. We therefore propose a model in which bioenergetic state is key to the physiological (and likely some of the pathophysiological) consequences of mitochondrial shape changes.

The simplest interpretation of our calcium manipulation experiments is that elongated and hyperpolarized mitochondria act as more efficient calcium sinks, with cytosolic calcium levels impacting dendrite and synapse development independently and in opposite directions. Indeed, dendritic spine development in hippocampal culture requires excitatory calcium influx (Kossel et al., 1997), but calcium influx can also promote growth cone collapse and neurite retraction (Gomez and Spitzer, 2000). In support of the notion that shape determines mitochondrial calcium buffering capacity, a previous report showed that inhibition of Drp1 enhances calcium uptake into mitochondria of cortical neurons (Saotome et al., 2008). At the other extreme, mitochondrial fragmentation via Fis1 overexpression was found to attenuate mitochondrial calcium uptake in HeLa cells (Frieden et al., 2004). However, $\Delta \Psi_{\mathrm{m}}$ can also influence calcium homeostasis indirectly through ATP or ROS production. For example, the activity of the plasma membrane $\mathrm{Na}^{+} / \mathrm{K}^{+}$exchanger and several calcium pumps is limited by ATP levels (Nicholls et al., 2003; Brini and Carafoli, 2009).

In addition to mitochondrial dynamics influencing calcium homeostasis, calcium can promote mitochondrial fragmentation by calcineurin-mediated dephosphorylation of Drp1 (Cribbs and 
A
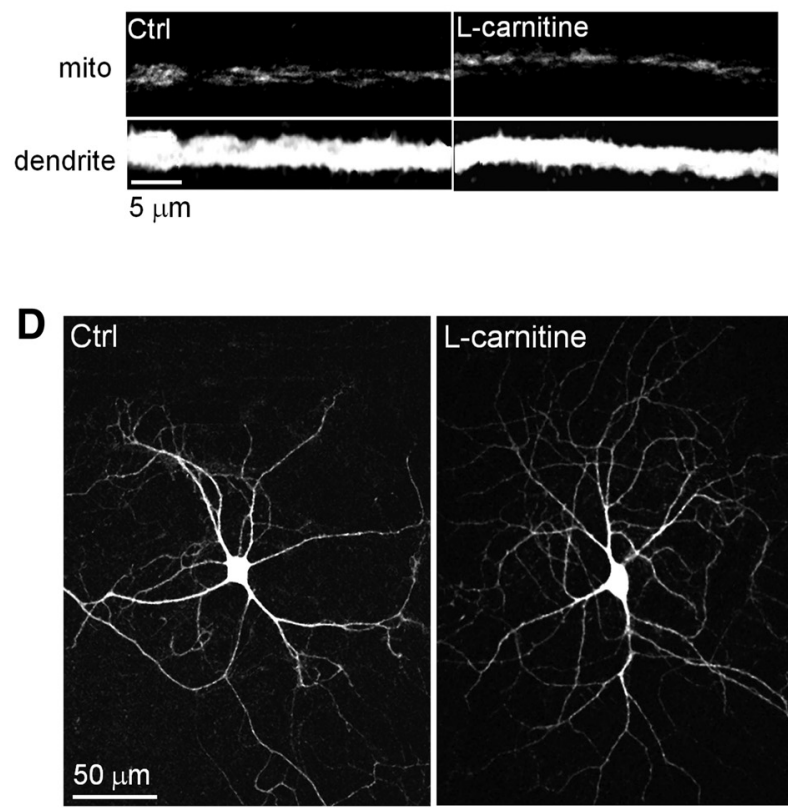

F

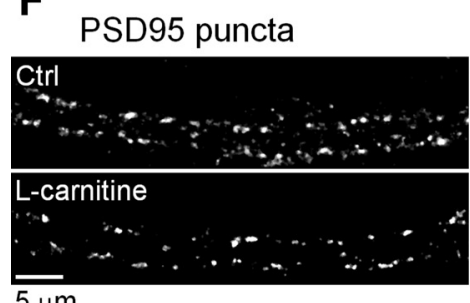

$5 \mu \mathrm{m}$
B

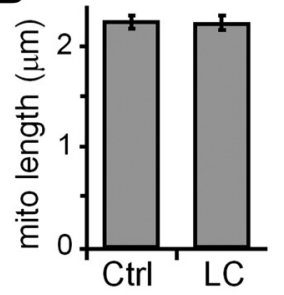

C

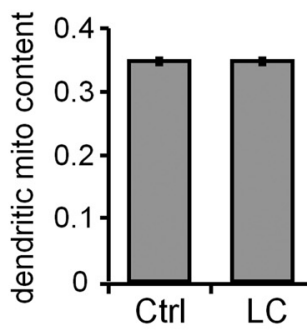

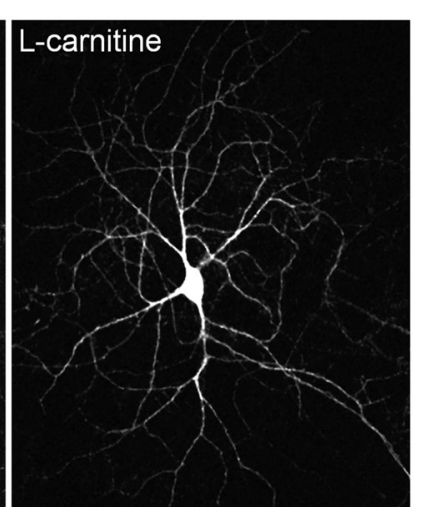

G

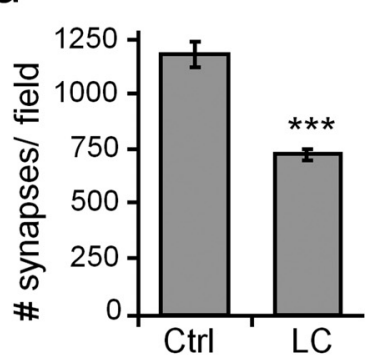

E

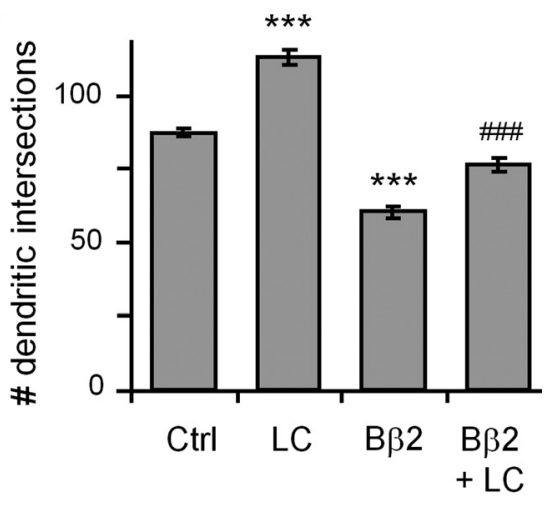

H

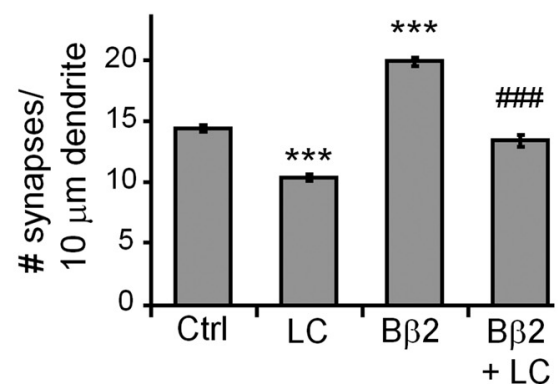

Figure 8. L-Carnitine phenocopies mitochondrial fusion effects on dendrite and synapse development. $\boldsymbol{A}-\boldsymbol{H}$, At 12 DIV, hippocampal neurons were virally transduced with GFP or B $\beta 2$-GFP and treated with or without L-carnitine (LC) (1 mM) starting at 13 DIV. At 18 DIV, cultures were fixed and stained for mitochondria (mito, TOM20; $\boldsymbol{A}--\boldsymbol{C}$ ), dendrites (MAP2B; $\boldsymbol{D}, \boldsymbol{E}$ ), and synapses (PSD95, $\boldsymbol{F}-\boldsymbol{H})$. Shown are representative images $(\boldsymbol{A}, \boldsymbol{D}, \boldsymbol{F})$ and quantification of mitochondrial length $(\boldsymbol{B})$, dendritic mitochondria content $(\boldsymbol{C}$ ), dendrite complexity $(\boldsymbol{E})$, synapse number (within a $318 \times 318 \mu \mathrm{m}$ visual field; $\boldsymbol{G})$, and synapse density $(\boldsymbol{H})$ plotting; means \pm SE of three experiments with $36+$ neurons/condition; ${ }^{* * *} p<0.001$ compared to untreated controls (Ctrl); ${ }^{\# \# \# ~} p<0.001$ compared to B $\beta 2$.

Strack, 2007; Cereghetti et al., 2008; Wang et al., 2011). Indeed, inhibiting calcineurin with FK506 or blocking spontaneous excitatory synaptic activity by TTX and APV treatment raises Drp1 Ser656 phosphorylation levels in hippocampal cultures (data not shown), implicating calcium/calcineurin in feedback amplification of $\mathrm{PP} 2 \mathrm{~A} / \mathrm{B} \beta 2$-mediated mitochondrial fission.

It is thought that neurons rely on efficient transport of mitochondria to synapses distant from the cell body to supply ATP for synaptic vesicle recycling and other energetically expensive processes (Hollenbeck and Saxton, 2005; Zinsmaier et al., 2009; MacAskill and Kittler, 2010). Furthermore, disturbance of the mitochondrial fission/fusion balance toward either extreme can interfere with mitochondrial transport, leading to organelle accumulation in the soma (Chen and Chan, 2009). Lack of fission can sterically prevent highly interconnected mitochondrial masses from entering into small caliber axons and dendrites. On the other hand, small mitochondria resulting from impaired fusion were observed to display mainly undirected Brownian motion, presumably because they do not efficiently attach to microtubules and other cytoskeletal elements (Chen et al., 2003). Indeed, failure of mitochondria to reach distant synaptic terminals may contribute to the pathogenesis of Charcot Marie Tooth disease type 2A, a common peripheral neuropathy caused by hypomorphic mutations in the
OMM fusion GTPase mitofusin-2 that affects the longest axons in the human body (Cartoni and Martinou, 2009).

However, simple ATP diffusion and high energy phosphate relay systems such as the phosphocreatine shuttle may obviate the need for synaptic mitochondria, at least in CNS neurons with shorter processes (Andres et al., 2008; Linton et al., 2010). In the hippocampal CA1 region, for instance, about $60 \%$ of presynaptic terminals are devoid of mitochondria (Shepherd and Harris, 1998). In addition, except for rare examples (e.g., the giant postsynaptic specializations on CA3 neurons that receive mossy fiber input from the dentate gyrus), postsynaptic dendritic spines in the vertebrate CNS generally do not contain mitochondria (Adams and Jones, 1982; Cameron et al., 1991; Chicurel and Harris, 1992). Lastly, motor nerve terminals in flies carrying mutations in Drp1 or dMiro, a mitochondrial transport protein, lack mitochondria, yet neuromuscular transmission deficits are only apparent after long or intense stimulation (Guo et al., 2005; Verstreken et al., 2005).

The effects of mitochondrial dynamics on synapse development described in this report do not support a critical role for dendritic localization of mitochondria. For one, we found that mitochondrial content in dendrites and synapse number are negatively correlated (e.g., Drp1 phosphorylation recruited mi- 


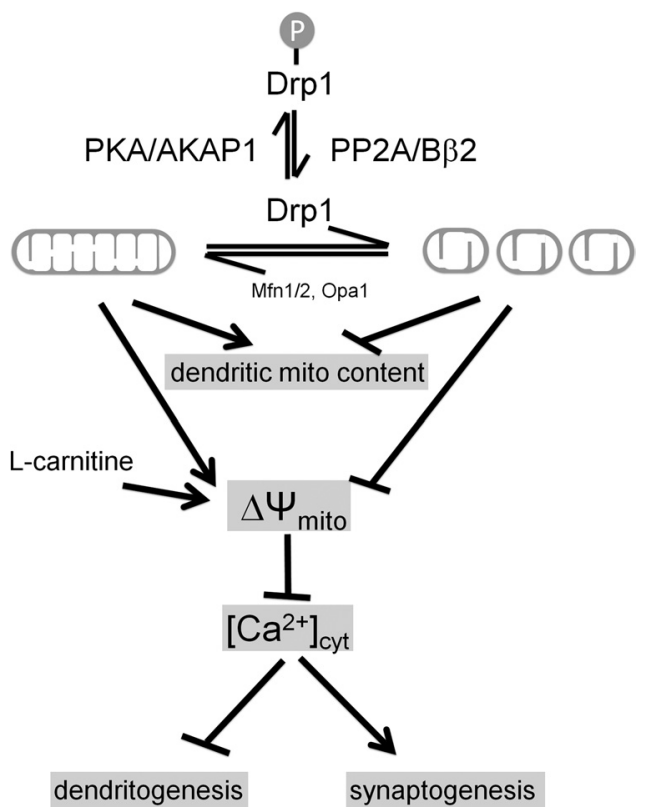

Figure 9. Model. Reversible phosphorylation of Drp1 at Ser656 by PKA/AKAP1 and PP2A/ $B \beta 2$ regulates neuronal development through mitochondrial (mito) membrane potential and calcium sequestration. See Discussion for details.

tochondria into dendrites but decreased synapse number). Secondly, fueling mitochondrial metabolism with L-carnitine inhibited synaptogenesis independently of mitochondrial shape or distribution. Our results contrast with a previous report that attributed the prosynaptogenic effect of Drp1 overexpression to accumulation of mitochondria in dendrites and increased metabolic activity (Li et al., 2004). While we cannot fully account for these discrepancies, it should be noted that in the previous paper, wild-type Drp1 transfection was accompanied by apparent fusion of dendritic mitochondria, while dominant-negative inhibition of Drp1 resulted in the appearance of punctiform, swollen mitochondria in dendrites (Li et al., 2004, their Fig. 4). Experimental manipulations that selectively modulate mitochondrial distribution (but not shape or function) are needed to resolve the relationship between mitochondrial transport and synapse development and function.

How do elongated mitochondria sustain higher metabolic activity? Gomes et al., recently reported that unopposed mitochondrial fusion due to PKA-mediated Drp1 phosphorylation increased oxygen consumption and ATP production in HeLa cells. Elongated mitochondria displayed higher cristae density and enhanced dimerization (and by inference, activity) of the ATP synthase (Gomes et al., 2011). Presumably, similar mechanisms operate in neurons.

Regulation of Drp1 by reversible phosphorylation is well documented in pathological contexts. For instance, PKA-mediated phosphorylation of Drp1 at Ser656 protects neuronal cells from various apoptotic insults (Cribbs and Strack, 2007; Merrill et al., 2011) and spares mitochondria from autophagic degradation (Gomes et al., 2011). Conversely, calcineurin-mediated dephosphorylation of Drp1 drives cardiomyocyte apoptosis during ischemia-reperfusion (Wang et al., 2011). The present report provides the first example of Drp1 phospho-regulation impinging on developmental processes. Important questions that need to be addressed concern the temporal and spatial regulation of the kinases and phosphatases that target Drp1 in neurons. For example, we neither understand the mechanisms by which PP2A/
$\mathrm{B} \beta 2$ gene induction occurs during postnatal brain development (Dagda et al., 2003), nor how this neuron-specific phosphatase dynamically shuttles between cytosol and the OMM (Dagda et al., 2008). In concert with the cAMP-PKA and calcium-calcineurin axes, regulated OMM translocation of $\mathrm{B} \beta 2$ may allow neurons to rapidly modify mitochondrial architecture to meet the changing demands for energy and calcium sequestration in the developing and mature CNS.

\section{References}

Adams I, Jones DG (1982) Quantitative ultrastructural changes in rat cortical synapses during early-, mid- and late-adulthood. Brain Res 239:349-363.

Affaitati A, Cardone L, de Cristofaro T, Carlucci A, Ginsberg MD, Varrone S, Gottesman ME, Avvedimento EV, Feliciello A (2003) Essential role of A-kinase anchor protein 121 for cAMP signaling to mitochondria. J Biol Chem 278:4286-4294.

Andres RH, Ducray AD, Schlattner U, Wallimann T, Widmer HR (2008) Functions and effects of creatine in the central nervous system. Brain Res Bull 76:329-343.

Baloh RH, Schmidt RE, Pestronk A, Milbrandt J (2007) Altered axonal mitochondrial transport in the pathogenesis of Charcot-Marie-Tooth disease from mitofusin 2 mutations. J Neurosci 27:422-430.

Bremer J (1983) Carnitine-metabolism and functions. Physiol Rev 63: $1420-1480$

Brini M, Carafoli E (2009) Calcium pumps in health and disease. Physiol Rev 89:1341-1378.

Cameron HA, Kaliszewski CK, Greer CA (1991) Organization of mitochondria in olfactory bulb granule cell dendritic spines. Synapse 8:107-118.

Carlucci A, Lignitto L, Feliciello A (2008) Control of mitochondria dynamics and oxidative metabolism by CAMP, AKAPs and the proteasome. Trends Cell Biol 18:604-613.

Cartoni R, Martinou JC (2009) Role of mitofusin 2 mutations in the physiopathology of Charcot-Marie-Tooth disease type 2A. Exp Neurol 218:268-273

Cereghetti GM, Stangherlin A, Martins de Brito O, Chang CR, Blackstone C, Bernardi P, Scorrano L (2008) Dephosphorylation by calcineurin regulates translocation of Drp1 to mitochondria. Proc Natl Acad Sci U S A 105:15803-15808.

Chang CR, Blackstone C (2007) Cyclic AMP-dependent protein kinase phosphorylation of Drp1 regulates its GTPase activity and mitochondrial morphology. J Biol Chem 282:21583-21587.

Chen H, Chan DC (2009) Mitochondrial dynamics-fusion, fission, movement, and mitophagy-in neurodegenerative diseases. Hum Mol Genet 18:R169-R176.

Chen H, Detmer SA, Ewald AJ, Griffin EE, Fraser SE, Chan DC (2003) Mitofusins Mfn1 and Mfn2 coordinately regulate mitochondrial fusion and are essential for embryonic development. J Cell Biol 160:189-200.

Chen Y, Stevens B, Chang J, Milbrandt J, Barres BA, Hell JW (2008) NS21: re-defined and modified supplement B27 for neuronal cultures. J Neurosci Methods 171:239-247.

Chicurel ME, Harris KM (1992) Three-dimensional analysis of the structure and composition of CA3 branched dendritic spines and their synaptic relationships with mossy fiber boutons in the rat hippocampus. J Comp Neurol 325:169-182.

Cribbs JT, Strack S (2007) Reversible phosphorylation of Drp1 by cyclic AMP-dependent protein kinase and calcineurin regulates mitochondrial fission and cell death. EMBO Rep 8:939-944.

Cribbs JT, Strack S (2009) Functional characterization of phosphorylation sites in dynamin-related protein 1. Methods Enzymol 457:231-253.

Dagda RK, Zaucha JA, Wadzinski BE, Strack S (2003) A developmentally regulated, neuron-specific splice variant of the variable subunit B-beta targets protein phosphatase $2 \mathrm{~A}$ to mitochondria and modulates apoptosis. J Biol Chem 278:24976-24985.

Dagda RK, Barwacz CA, Cribbs JT, Strack S (2005) Unfolding-resistant translocase targeting: A novel mechanism for outer mitochondrial membrane localization exemplified by the $\mathrm{B} \beta 2$ regulatory subunit of protein phosphatase 2A. J Biol Chem 280:27375-27382.

Dagda RK, Merrill RA, Cribbs JT, Chen Y, Hell JW, Usachev YM, Strack S (2008) The spinocerebellar ataxia 12 gene product and protein phospha- 
tase $2 \mathrm{~A}$ regulatory subunit Bbeta2 antagonizes neuronal survival by promoting mitochondrial fission. J Biol Chem 283:36241-36248.

Farkas DL, Wei MD, Febbroriello P, Carson JH, Loew LM (1989) Simultaneous imaging of cell and mitochondrial membrane potentials. Biophys J 56:1053-1069.

Frieden M, James D, Castelbou C, Danckaert A, Martinou JC, Demaurex N (2004) $\mathrm{Ca}\left({ }^{2+}\right)$ homeostasis during mitochondrial fragmentation and perinuclear clustering induced by hFis1. J Biol Chem 279:22704-22714.

Gomes LC, Di Benedetto G, Scorrano L (2011) During autophagy mitochondria elongate, are spared from degradation and sustain cell viability. Nat Cell Biol 13:589-598.

Gomez TM, Spitzer NC (2000) Regulation of growth cone behavior by calcium: new dynamics to earlier perspectives. J Neurobiol 44:174-183.

Guo X, Macleod GT, Wellington A, Hu F, Panchumarthi S, Schoenfield M, Marin L, Charlton MP, Atwood HL, Zinsmaier KE (2005) The GTPase dMiro is required for axonal transport of mitochondria to Drosophila synapses. Neuron 47:379-393.

Hollenbeck PJ, Saxton WM (2005) The axonal transport of mitochondria. J Cell Sci 118:5411-5419.

Holmes SE, O'Hearn EE, McInnis MG, Gorelick-Feldman DA, Kleiderlein JJ, Callahan C, Kwak NG, Ingersoll-Ashworth RG, Sherr M, Sumner AJ, Sharp AH, Ananth U, Seltzer WK, Boss MA, Vieria-Saecker AM, Epplen JT, Riess O, Ross CA, Margolis RL (1999) Expansion of a novel CAG trinucleotide repeat in the $5^{\prime}$ region of PPP2R2B is associated with SCA12 [letter]. Nat Genet 23:391-392.

Ishihara N, Nomura M, Jofuku A, Kato H, Suzuki SO, Masuda K, Otera H, Nakanishi Y, Nonaka I, Goto Y, Taguchi N, Morinaga H, Maeda M, Takayanagi R, Yokota S, Mihara K (2009) Mitochondrial fission factor Drp1 is essential for embryonic development and synapse formation in mice. Nat Cell Biol 11:958-966.

Janssens V, Longin S, Goris J (2008) PP2A holoenzyme assembly: in cauda venenum (the sting is in the tail). Trends Biochem Sci 33:113-121.

Kossel AH, Williams CV, Schweizer M, Kater SB (1997) Afferent innervation influences the development of dendritic branches and spines via both activity-dependent and non-activity-dependent mechanisms. J Neurosci 17:6314-6324.

Lackner LL, Nunnari JM (2009) The molecular mechanism and cellular functions of mitochondrial division. Biochim Biophys Acta 1792:11381144.

Li H, Chen Y, Jones AF, Sanger RH, Collis LP, Flannery R, McNay EC, Yu T, Schwarzenbacher R, Bossy B, Bossy-Wetzel E, Bennett MV, Pypaert M, Hickman JA, Smith PJ, Hardwick JM, Jonas EA (2008) Bcl-xL induces Drp1-dependent synapse formation in cultured hippocampal neurons. Proc Natl Acad Sci U S A 105:2169-2174.

Li Z, Sheng M (2003) Some assembly required: the development of neuronal synapses. Nat Rev Mol Cell Biol 4:833-841.

Li Z, Okamoto K, Hayashi Y, Sheng M (2004) The importance of dendritic mitochondria in the morphogenesis and plasticity of spines and synapses. Cell 119:873-887.

Lim IA, Merrill MA, Chen Y, Hell JW (2003) Disruption of the NMDA receptor-PSD-95 interaction in hippocampal neurons with no obvious physiological short-term effect. Neuropharmacology 45:738-754.

Lin RY, Moss SB, Rubin CS (1995) Characterization of S-AKAP84, a novel developmentally regulated A kinase anchor protein of male germ cells. J Biol Chem 270:27804-27811.

Linton JD, Holzhausen LC, Babai N, Song H, Miyagishima KJ, Stearns GW, Lindsay K, Wei J, Chertov AO, Peters TA, Caffe R, Pluk H, Seeliger MW, Tanimoto N, Fong K, Bolton L, Kuok DL, Sweet IR, Bartoletti TM, Radu RA, et al. (2010) Flow of energy in the outer retina in darkness and in light. Proc Natl Acad Sci U S A 107:8599-8604.

Livigni A, Scorziello A, Agnese S, Adornetto A, Carlucci A, Garbi C, Castaldo I, Annunziato L, Avvedimento EV, Feliciello A (2006) Mitochondrial AKAP121 links cAMP and src signaling to oxidative metabolism. Mol Biol Cell 17:263-271.

MacAskill AF, Kittler JT (2010) Control of mitochondrial transport and localization in neurons. Trends Cell Biol 20:102-112.

Merrill RA, Dagda RK, Dickey AS, Cribbs JT, Green SH, Usachev YM, Strack S (2011) Mechanism of neuroprotective mitochondrial remodeling by PKA/AKAP1. PLoS Biol 9:e1000612.

Misko A, Jiang S, Wegorzewska I, Milbrandt J, Baloh RH (2010) Mitofusin 2 is necessary for transport of axonal mitochondria and interacts with the Miro/Milton complex. J Neurosci 30:4232-4240.
Nicholls DG, Vesce S, Kirk L, Chalmers S (2003) Interactions between mitochondrial bioenergetics and cytoplasmic calcium in cultured cerebellar granule cells. Cell Calcium 34:407-424.

O’Brien RJ, Kamboj S, Ehlers MD, Rosen KR, Fischbach GD, Huganir RL (1998) Activity-dependent modulation of synaptic AMPA receptor accumulation. Neuron 21:1067-1078.

Otera H, Mihara K (2011) Molecular mechanisms and physiologic functions of mitochondrial dynamics. J Biochem 149:241-251.

Perrino C, Feliciello A, Schiattarella GG, Esposito G, Guerriero R, Zaccaro L, Del Gatto A, Saviano M, Garbi C, Carangi R, Di Lorenzo E, Donato G, Indolfi C, Avvedimento VE, Chiariello M (2010) AKAP121 downregulation impairs protective cAMP signals, promotes mitochondrial dysfunction, and increases oxidative stress. Cardiovasc Res 88:101-110.

Pivovarova NB, Andrews SB (2010) Calcium-dependent mitochondrial function and dysfunction in neurons. FEBS J 277:3622-3636.

Rao A, Craig AM (1997) Activity regulates the synaptic localization of the NMDA receptor in hippocampal neurons. Neuron 19:801-812.

Romanello V, Guadagnin E, Gomes L, Roder I, Sandri C, Petersen Y, Milan G, Masiero E, Del Piccolo P, Foretz M, Scorrano L, Rudolf R, Sandri M (2010) Mitochondrial fission and remodelling contributes to muscle atrophy. EMBO J 29:1774-1785.

Saotome M, Safiulina D, Szabadkai G, Das S, Fransson A, Aspenstrom P, Rizzuto R, Hajnóczky G (2008) Bidirectional $\mathrm{Ca}^{2+}$-dependent control of mitochondrial dynamics by the Miro GTPase. Proc Natl Acad Sci U S A 105:20728-20733.

Scaduto RC Jr, Grotyohann LW (1999) Measurement of mitochondrial membrane potential using fluorescent rhodamine derivatives. Biophys J 76:469-477.

Schmidt K, Kins S, Schild A, Nitsch RM, Hemmings BA, Götz J (2002) Diversity, developmental regulation and distribution of murine PR55/B subunits of protein phosphatase 2A. Eur J Neurosci 16:2039-2048.

Shepherd GM, Harris KM (1998) Three-dimensional structure and composition of $\mathrm{CA3}->\mathrm{CA} 1$ axons in rat hippocampal slices: implications for presynaptic connectivity and compartmentalization. J Neurosci 18:8300-8310.

Turrigiano GG, Leslie KR, Desai NS, Rutherford LC, Nelson SB (1998) Activity-dependent scaling of quantal amplitude in neocortical neurons. Nature 391:892-896.

Vaillant AR, Zanassi P, Walsh GS, Aumont A, Alonso A, Miller FD (2002) Signaling mechanisms underlying reversible, activity-dependent dendrite formation. Neuron 34:985-998.

Van Aelst L, Cline HT (2004) Rho GTPases and activity-dependent dendrite development. Curr Opin Neurobiol 14:297-304.

Verburg J, Hollenbeck PJ (2008) Mitochondrial membrane potential in axons increases with local nerve growth factor or semaphorin signaling. J Neurosci 28:8306-8315.

Verstreken P, Ly CV, Venken KJ, Koh TW, Zhou Y, Bellen HJ (2005) Synaptic mitochondria are critical for mobilization of reserve pool vesicles at Drosophila neuromuscular junctions. Neuron 47:365-378.

Wakabayashi J, Zhang Z, Wakabayashi N, Tamura Y, Fukaya M, Kensler TW, Iijima M, Sesaki H (2009) The dynamin-related GTPase Drp1 is required for embryonic and brain development in mice. J Cell Biol 186:805-816.

Wang JX, Jiao JQ, Li Q, Long B, Wang K, Liu JP, Li YR, Li PF (2011) miR499 regulates mitochondrial dynamics by targeting calcineurin and dynamin-related protein-1. Nat Med 17:71-78.

Waterham HR, Koster J, van Roermund CW, Mooyer PA, Wanders RJ, Leonard JV (2007) A lethal defect of mitochondrial and peroxisomal fission. N Engl J Med 356:1736-1741.

Westermann B (2010) Mitochondrial fusion and fission in cell life and death. Nat Rev Mol Cell Biol 11:872-884.

Yano H, Oyanagi E, Kato Y, Samejima Y, Sasaki J, Utsumi K (2010) $\mathrm{L}$-carnitine is essential to beta-oxidation of quarried fatty acid from mitochondrial membrane by PLA(2). Mol Cell Biochem 342:95-100.

Yoon Y, Pitts KR, Dahan S, McNiven MA (1998) A novel dynamin-like protein associates with cytoplasmic vesicles and tubules of the endoplasmic reticulum in mammalian cells. J Cell Biol 140:779-793.

Zha XM, Green SH, Dailey ME (2005) Regulation of hippocampal synapse remodeling by epileptiform activity. Mol Cell Neurosci 29:494-506.

Zinsmaier KE, Babic M, Russo GJ (2009) Mitochondrial transport dynamics in axons and dendrites. Results Probl Cell Differ 48:107-139. 